\title{
BMJ Quality Improving the communication between teams managing boarded patients on a surgical specialty ward
}

\author{
Shobitha Puvaneswaralingam, Daniella Ross
}

\section{To cite:}

Puvaneswaralingam S, Ross D. Improving the communication between teams managing boarded patients on a surgical specialty ward. BMJ Quality Improvement Reports 2016;5:u209186.w3750. doi:10.1136/bmjquality. u209186.w3750

Received 25 November 2015 Revised 13 July 2016 Published Online First 21 July 2016

CrossMark

NHS Tayside, United Kingdom

\section{Correspondence to} Shobitha Puvaneswaralingam spuvaneswaralingam@nhs. net

\section{ABSTRACT}

Transferring patients from the ward of their specialty or consultant is described as boarding. ${ }^{1}$ Boarding patients is becoming increasingly prevalent due to greater pressure on hospital capacity. This practice compromises patient safety through delayed investigations, prolonged hospital stays, and increased risk of hospital-acquired infections. ${ }^{12}$ We evaluated how regularly boarded patients were reviewed, and how effectively information regarding their management was communicated from their primary specialty to ward staff. We aimed to improve the frequency of patient reviews by ensuring that each patient was reviewed every weekday and increase communication between primary specialty, and medical and nursing teams by $20 \%$ from baseline during the data collection period.

The project was based in the Otolaryngology ward in Ninewells Hospital, Dundee, where there was a high prevalence of boarded patients. Baseline data showed a clear deficit in communication between the primary specialty and ward staff with only $31 \%$ of patient reviews being communicated to ward doctors. We designed and implemented a communication tool, in the form of a sticker, to be inserted into patients' medical notes for use by the primary specialty.

Implementation of the sticker improved communication between teams as stickers were completed in $93 \%$ of instances. In $88 \%$ of patient reviews, the junior doctor was informed of the management plan, showing a large increase from baseline. Through PDSA cycles, we aimed to increase the sustainability and reliability of the sticker; however, we faced challenges with sustainability of sticker insertion. We aim to engage more stakeholders to raise awareness of the problem, brainstorm solutions together, and review the production and implementation of stickers with senior hospital management to discuss the potential use of this tool within practice. There is potentially a large scope for utilisation of this communication tool on a local level, which we hope will significantly improve patient safety.

\section{PROBLEM}

Transferring patients from the ward of their specialty or consultant is described as boarding. Boarding of patients has become more prevalent within the NHS in order to improve patient flow. ${ }^{1}$ There are numerous factors contributing to the boarding of patients, including increased admissions, inadequate resources, reduced levels of staffing, and poor collaboration between health and social care. ${ }^{1}$

It is well known that boarding of patients compromises patient safety. ${ }^{3}{ }^{4}$ This is due to the loss of continuity in care as well as an increased length of stay which leads to compromised patient care. ${ }^{2}$ Boarding also contributes to poorer outcomes such as hospital acquired infection, delayed discharge, and readmission following discharge. ${ }^{12}$

In Scotland, junior doctors and nurses provide ward-based care whilst specialty doctors such as registrars and consultants, are firm-based. With ward-based care, staff manage patients within their ward irrespective of the patient's primary specialty. Firm-based care involves managing patients from a specific specialty within any ward. Communication between these teams is through ward-based paper medical notes.

Whilst working in a busy Otolaryngology ward in a tertiary teaching hospital in Dundee, Scotland with a high turnover of day-case patients, we as junior doctors identified that boarding of patients onto our ward was a common occurrence due to the high number of empty beds overnight. Boarding leads to problems such as delayed investigations, inadequate review by their primary team, and longer hospital stay as a result of poor communication. ${ }^{12}$

\section{BACKGROUND}

There has been increased pressure on hospital capacity nationwide in recent years. This is due to increased acute medical workload, greater pressure on doctors, and inefficient working practices. $^{2}{ }^{4}$ There are increasing financial pressures on the National Health Service (NHS) and as each 
occupied bed comes with a cost implication, there is little incentive to increase bed capacity. ${ }^{5}$ All hospitals in Scotland rely on the boarding of patients to ease the winter bed pressures. This refers to the seasonal increase in morbidity and mortality secondary to cold weather and the dangers of snow and ice. ${ }^{6} 7$ On a local level, boarding has been a solution to increased demand for unscheduled care, increased pressure on social care systems and failure to fill rota vacancies. ${ }^{8-10}$ Our experiences stem from working in a busy surgical specialty within a tertiary teaching centre, Ninewells Hospital, where patients are often boarded to aid patient flow. ${ }^{8}$

The frequent boarding of patients on our surgical specialty ward led to a number of pitfalls. On a frequent basis we noted that patients were not reviewed regularly, stayed in hospital for an extra length of time, and were unsuitably managed by staff who were unfamiliar with specific conditions pertaining to the patient. When patients are boarded, there is often poor timing of visits from the primary specialty. The nurse or junior doctor looking after the patient was often absent from the ward at the time of the ward visit, hindering effective communication. This also impacted on communication with relatives. There was difficulty in obtaining adequate reviews by allied health professionals (AHPs) who felt knowledgeable enough to manage boarded patients, as AHPs on our surgical specialty ward were well-trained in conditions relating to our specific surgical specialty. ${ }^{8}$ A large concern was the inappropriate boarding of sick or unstable patients who required more input from the primary specialty. Another concern was that boarded patients may have felt that they received less attention than other patients due to irregular reviews. ${ }^{5}$

Boarding of patients continues to create issues in many hospitals across Scotland and a report from the winter bed pressures in 2009 strongly advised for the elimination of the practice of boarding. ${ }^{6}$ The boarding of patients within Ninewells Hospital has already been highlighted as a patient safety issue through a previous audit. This audit emphasised the importance of communication between specialties and good documentation. Interventions at this time included colour-coding patients' names on the ward board and leaving a reminder note in front of patients' case notes. Despite showing initial improvements to communication and documentation, this was not sustained. ${ }^{11}$ Other solutions have been proposed including having a large single flexible shared specialty area, consultant-led care, and wider availability of specialty-specific staff. ${ }^{5}$

\section{BASELINE MEASUREMENT}

Baseline data was prospectively collected over a 10-day period, excluding weekends, at the end of April 2015 in the Otolaryngology ward, Ninewells Hospital, Dundee. Weekends were excluded due to the irregularity of patient reviews out of hours. We planned to evaluate how regularly patients were reviewed and followed up, as well as how effectively information regarding their management plan was communicated from the primary specialty to the ward staff. We aimed to improve the frequency of patient review by ensuring that every patient was reviewed each weekday during the data collection period of nine days. We also aimed to increase communication of management plans from primary specialty to both medical and nursing teams by $20 \%$ from baseline.

We noted the patient's primary specialty, whether they were reviewed, whether there were outstanding investigations, blood tests or discharge letters, whether a referral to another specialty was needed, and whether the junior doctors or nurses were informed of the management plan for the patient.

There were seven different specialties who boarded patients to the Otolaryngology ward during the baseline data collection, all of which were medical specialties. These included General Internal Medicine, Infectious Diseases, Gastroenterology, Respiratory, Rheumatology, Cardiology, and Neurology. On one occasion, this resulted in patients boarding from five different specialties in one day.

Over the 10-day period, there were 15 individual patients boarded to the ward. There was always a minimum of two boarding patients each day over the monitoring period and on one occasion there were eight different boarding patients on the ward. Throughout the monitoring period, there were nine occasions where patients were not reviewed by their primary specialty. One patient was on the ward for eight days and was only reviewed four times during their stay.

In 29 out of 42 patient reviews $(69 \%)$, the junior doctor on the ward was not informed of the management plan for the patient and in 17 out of 42 patient reviews $(40 \%)$, the nursing team were not informed. Of the 29 patient reviews that were not communicated to the junior doctor, four patients had outstanding investigations, five had outstanding blood tests, one patient required a referral to another specialty, and six patients required a discharge letter allowing them to go home.

\section{DESIGN}

The baseline data collection showed that there was a clear deficit in communication between primary specialties and staff caring for boarding patients, as well as infrequent reviews of boarded patients. In order to improve the management of boarded patients, we aimed to implement a sustainable and reliable tool to improve communication.

Discussion with the patient safety lead for the department highlighted previous research on this topic. Previous methods to tackle this problem included a reminder note placed in front of the boarded patient's case notes as well as highlighting the names of boarded patients on the ward board in order to increase awareness. ${ }^{11}$ After consultation with ward staff, we decided to 
Figure 1 Sticker

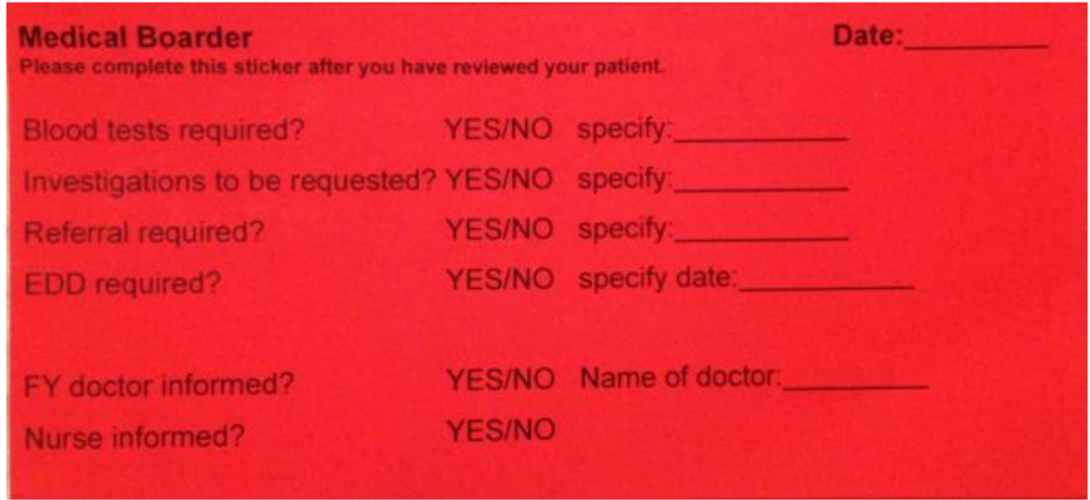

create a sticker which would provide an easy communication tool to transfer key information regarding the patient's management between staff. The sticker would be inserted by the junior doctor on the ward into the patient's notes prior to review and would be completed each day by their primary specialty after the patient was reviewed.

The outcome measures of our project evaluated how often boarded patients were reviewed by their primary specialty. The process measures examined how well information regarding the patient's management plan was communicated from the patient's primary speciality to the ward staff. Balancing measures included patient satisfaction with their care and the effect on the length of hospital stay; however these were not explicitly measured.

The sticker was designed with key points in mind as gained from the baseline data collection. With regards to content, we included specific points related to the patient's management including outstanding investigations and blood tests, referrals to specialties, and pending discharge letters. The formatting of the A7 sticker was simple and eye-catching, as it was bright orange with points to circle "yes" or "no" answers. The sticker was further developed to include free text to specify more detailed information as well as the name of the doctor or nurse informed (see Figure 1). A bright orange sign accompanied the stickers and was placed on the notes trolley specific to boarded patients (see Figure 2).

Stickers are a low-cost and effective tool which can easily be reproduced in all departments and can be modified or updated for each specialty. The ultimate goal would be for all specialties who board out patients to utilise these stickers and this would be integrated into routine practice across the NHS board.

\section{STRATEGY}

PDSA cycle 1: Improving communication

We aimed to improve the frequency of patient reviews by ensuring that every patient was reviewed every weekday during the data collection period. We also aimed to increase communication of management plans from

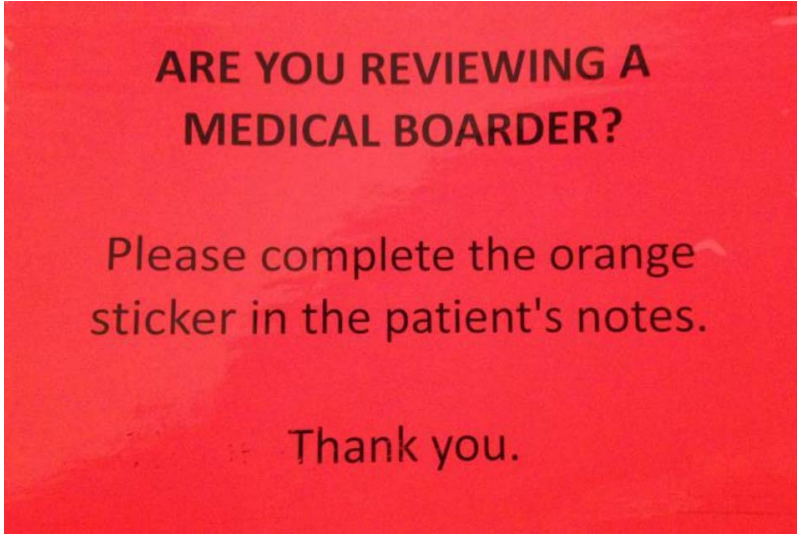

Figure 2 Notes Trolley Sign

primary specialty to both medical and nursing teams by $20 \%$ from baseline during our data collection period. We implemented a communication tool, in the form of a sticker inserted into boarded patients' medical notes, which demonstrated improved frequency of patient reviews with 42 of 43 patients being reviewed. Stickers were completed for 39 patient reviews. Communication between teams improved as the medical staff were informed of patient management plans in 37 out of 42 $(88 \%)$ occasions and nursing staff were informed in 36 out of $42(86 \%)$ of occasions.

PDSA cycle 2: Improving reliability and sustainability

We aimed to create a reliable and sustainable process in which stickers were produced and inserted into the boarded patient's medical notes for each patient review during the data collection period. We created a stand containing a supply of stickers with advice to their insertion to serve as a prompt for visiting teams. Of the five patient reviews during the period of data collection, all patients were reviewed by their primary specialty. However, only one sticker was inserted and completed by the primary specialty, which was communicated to the junior doctor and nurse.

Discussions with the senior charge nurse and secretarial staff highlighted concerns that sticker production and implementation would increase their workload. A sustainable method of sticker production could not be identified using these teams. 
See supplementary file: ds10726.docx - "Supplementary material- PDSA Cycles"

\section{RESULTS}

Following the implementation of our intervention, data was prospectively collected over a nine-day period in the Otolaryngology ward. There were four different specialities who boarded patients to our ward including three medical specialties and one general surgical specialty. On certain days during this period, all four specialties had patients boarded to the ward. Over the nine-day period, there were 17 individual patients boarded to the ward, with a minimum of one boarded patient each day. The maximum number of boarded patients on the ward on a single day was nine.

Of the 43 potential patient reviews, one patient was not reviewed by their primary specialty although the patient required a discharge letter. On four (10\%) occasions, a sticker was not completed following patient review. One of these patients required blood tests; the medical team were not informed, however a nurse was told. Of the four stickers not completed, two patients required no changes to their current management plan. On occasions where stickers were completed, the junior doctor was informed of the management plan in 37 out of $42(88 \%)$ patient reviews, and the nursing staff were informed in 36 out of $42(86 \%)$ patient reviews (see Figures 3 and 4).

Following implementation of the sticker, we attempted to make the communication tool sustainable and reliable by encouraging the primary specialties to insert the sticker into patients' notes themselves prior to review. In order to simplify the process, we created a large stand containing stickers with advice to their insertion to serve as a prompt for visiting medical teams. Due to variation in the number of boarded patients, there were fewer patients on the ward during our data collection period of nine days. There were two different specialties who boarded patients to our ward and a total of five individual patients boarded to the ward during the nine-day data collection period. On five days, there were no boarded patients on the ward. During the remaining four days, there was a maximum of two patients in a single day. Out of the five potential patient reviews, over the nine-day period of data collection, all patients were reviewed by their primary specialty. However, only one sticker was completed by the primary specialty for a patient requiring blood tests and the junior doctor and nurse were informed of this. Of the four patient reviews where a sticker was not inserted nor completed, the medical and nursing teams were not informed of the management plans. Although, these patient reviews had no outstanding tasks.

With the aim of improving sustainability of sticker production and implementation, discussions were had with the senior charge nurse and secretarial staff on the Otolaryngology ward. Secretarial staff highlighted concerns that sticker production would increase their workload and hence advised a central production of stickers out with the ward. Nursing staff expressed further concerns that sticker implementation would add to their workload. A sustainable method of sticker production could not be identified using these methods.

\section{LESSONS AND LIMITATIONS}

Implementing our communication tool improved the handover of information between teams. In addition to this, feedback from staff has been positive in enabling

\section{Communication between primary specialty and junior doctor}

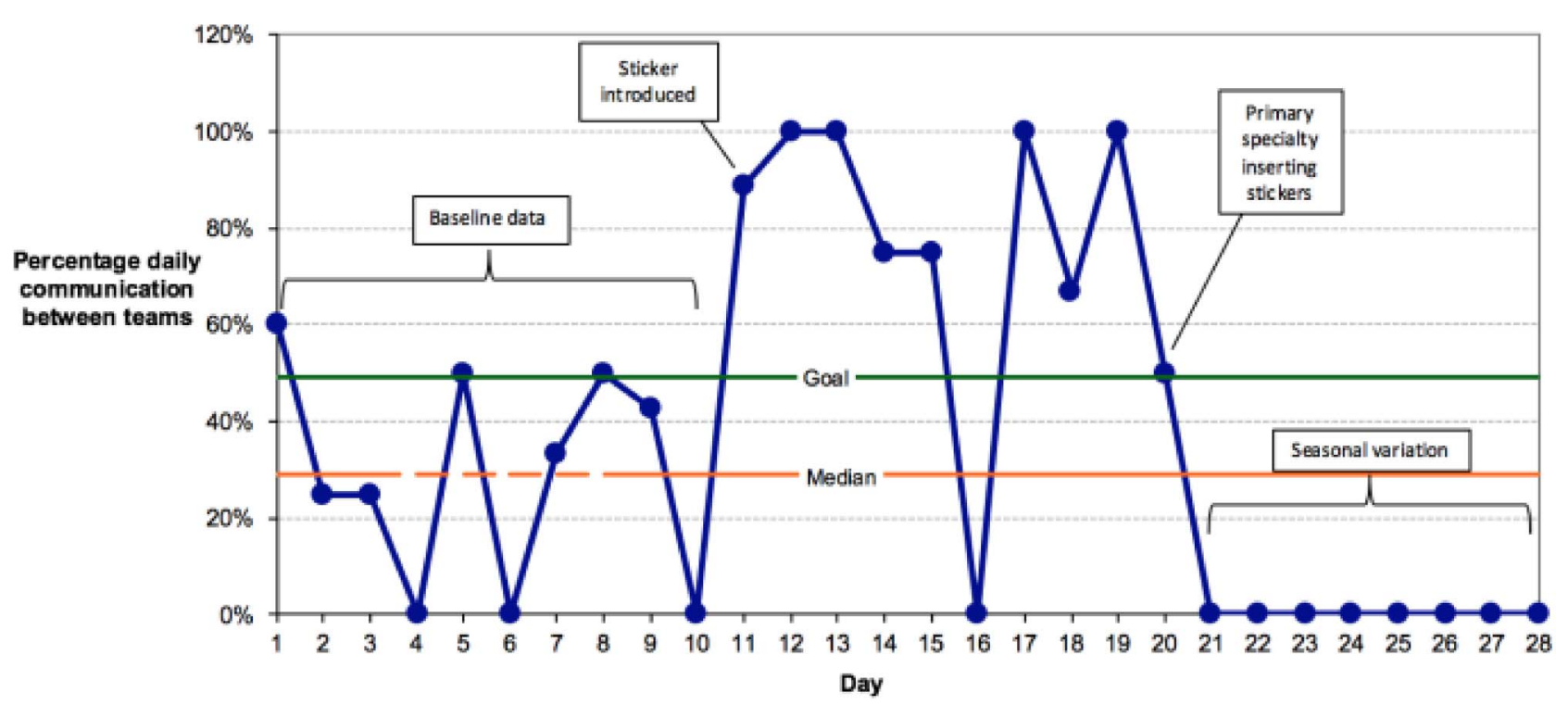

Figure 3 Run chart demonstrating percentage of communication between primary specialty and junior doctor 
Communication between primary specialty and nurse

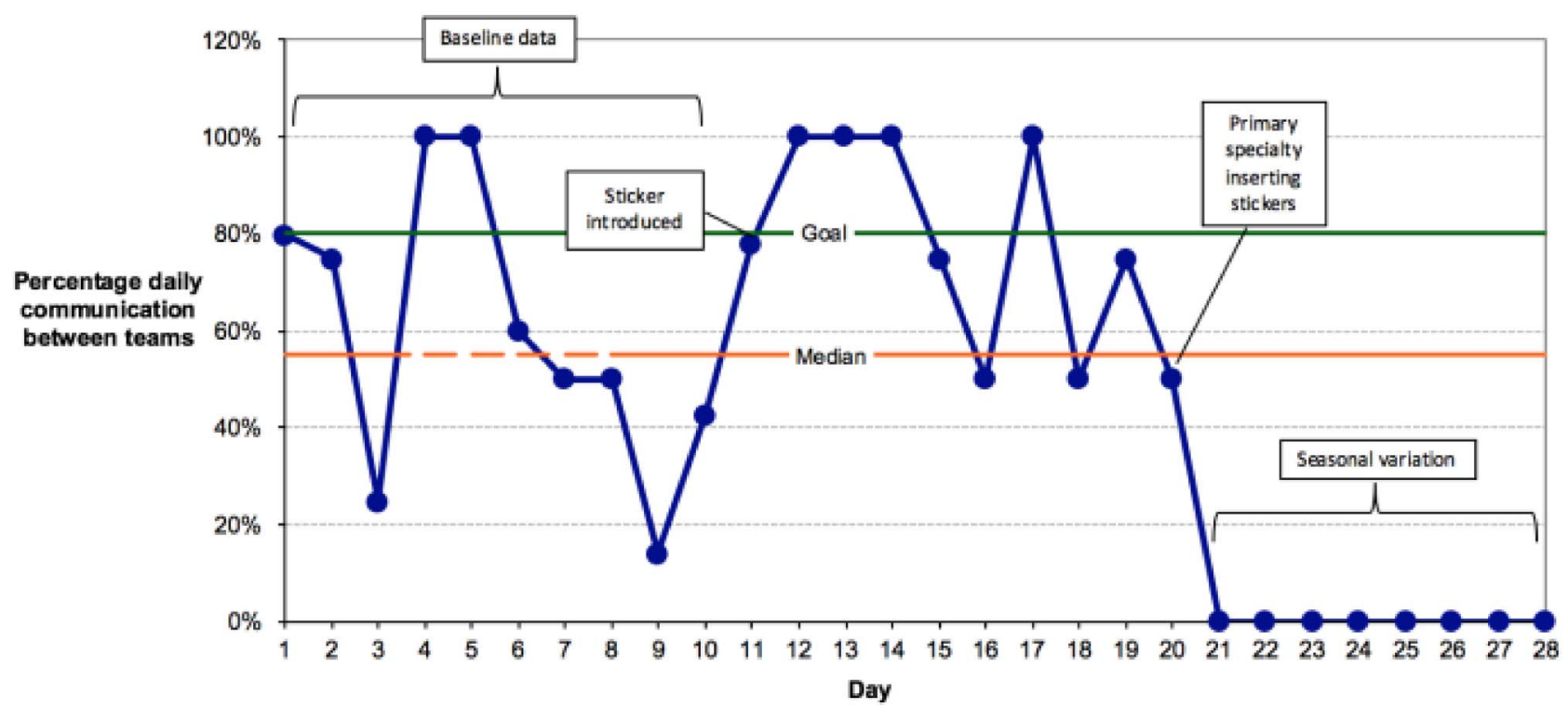

Figure 4 Run chart demonstrating percentage of communication between primary specialty and ward nurse

an efficient and simple way of transferring information regarding patient management plans; this is reflected in our data.

There were a number of limitations to this quality improvement project. The main challenge we faced was establishing a sustainable and reliable method of producing and inserting the stickers. The current method of ward-based junior doctors inserting stickers into patient notes prior to their review was not sustainable due to the quick turnover of junior staff. Attempts to encourage primary specialties to insert the stickers themselves proved to be unsuccessful. There was also a vast difference in sample size with each PDSA cycle due to reduced numbers of boarders in the ward, which was secondary to seasonal variation. Data was collected in the spring and summer when winter bed pressures were diminishing. This meant that we were not able to trial further changes to make the communication tool completely sustainable.

The engagement of stakeholders proved to be more challenging than we previously anticipated. Discussions with the senior charge nurse of the ward highlighted concerns from nursing staff that the responsibility of implementing stickers would fall to them. In order to make this tool truly sustainable, it would be preferable for the primary specialties to be responsible for the implementation of stickers for their patients. To ensure the sustainability of the communication tool, it is important to remove the influence of human factors, as specified above.

We also identified three measures that were not examined. The first outcome relates to evaluating patient satisfaction as a result of improved communication between primary specialties and the ward team caring for the patient. The project assessed communication and documentation between specialties however data pertaining to patient satisfaction with their care was not reviewed. Secondly we failed to measure the precise impact of improved communication on patient management. Our literature review demonstrated that the boarding of patients could lead to prolonged hospital stay and increase their risk of hospital acquired infection. On data collection, we failed to identify these outcomes and therefore we were unable to draw conclusions on the impact of boarding on patient management. Lastly, the cost implication of sticker production was not formally evaluated. There would be a small cost implication in the production of stickers; however if this led to reduced length of patient stay, the cost benefits would be favourable. Discussions with the ward assistant identified challenges in mass-producing stickers within a ward environment and advice was given to engage with more senior management to provide stickers from a central production service.

In order to further develop this quality improvement project, it would be favourable to trial this communication tool across other wards given our positive findings from one ward; however this will require a review of the root causes leading to potential adverse outcomes in the management of boarded patients. Analysis of the root causes should involve more stakeholders in order to brainstorm solutions that are more likely to be sustainable. In order to improve sustainability we need to increase awareness of the problem of boarding amongst staff, specifically primary specialties boarding patients and wards receiving boarded patients. We also aim to further develop this quality improvement project through repeat PDSA cycles, specifically examining the impact on management of boarded patients as well as collecting more data to demonstrate sustainability over 
time. On a national level, and in keeping with the Scottish Government's 6 Essential Actions to improve unscheduled care, this project has the potential scope to contribute towards these targets by providing data on current practice and by suggesting a tool for performance improvements. ${ }^{8}$ We recognise that there are limitations to the generalisability of this project due to the setting and process in which it took place. Other hospitals and ward settings may practice firm-based care, in which specialty-specific junior doctors would be involved in formulating management decisions for patients, as well as executing them. We also recognise that boarding is not common place outside of Scotland and that communication between teams may vary elsewhere, such as through the use of electronic records.

\section{CONCLUSION}

The management of boarded patients is a risk to patient safety as it prolongs hospital stay, delays investigations and is more likely to result in readmission following discharge. $^{2}$ We identified a vast deficit in communication between primary specialties and the ward team with regards to boarded patient management. We implemented a low-cost communication tool, in the form of a sticker, to aid the transfer of information between teams and improve the care of boarded patients. This proved to be highly successful as we demonstrated improved communication between specialties of boarded patients.

The communication tool was tested within one ward, with a small sample size, and showed some positive improvements. However, this project is still in its early stages and the aim is to trial it hospital-wide. Limitations of the project included a lack of sustainability in its current form alongside a deficit in reviewing balancing measures, specifically length of hospital stay, patient satisfaction and incidence of hospital acquired infections. We aim to engage more stakeholders to raise awareness of the problem and brainstorm solutions together, as well as review the production and implementation of stickers with senior hospital management to discuss the potential use of this tool within hospital practice. We also aim to further develop this quality improvement project through repeat PDSA cycles, specifically examining the impact on management of boarded patients to demonstrate sustainability over time. There is potentially a large scope for distribution and utilisation of this communication tool on both a local and national level, which we hope will significantly improve patient safety.

Acknowledgements Dr Sunny Jabbal, SCN Jane Park, Ashley Brown, Mr Sam Majumdar

Declaration of interests Nothing to declare.

Ethical approval The work being reported was deemed exempt from ethics review according to local policy.

Open Access This is an open-access article distributed under the terms of the Creative Commons Attribution Non-commercial License, which permits use, distribution, and reproduction in any medium, provided the original work is properly cited, the use is non commercial and is otherwise in compliance with the license. See:

- http://creativecommons.org/licenses/by-nc/2.0/

- http://creativecommons.org/licenses/by-nc/2.0/legalcode

\section{REFERENCES}

1. Beckett D. Boarding - Impact on patients, hospitals and healthcare systems. [Lecture] Society of Acute Medicine. May 2014. http://www. acutemedicine.org.uk/wp-content/uploads/2014/11/Plenary-5-1030Wrong-Place-Anytime-Why-Boarding-is-Bad-for-Patients-Hospitalsand-Healthcare-Systems.pdf (accessed November 19th 2015).

2. Royal College of Physicians of Edinburgh. Pressures in acute medical specialties. https://www.rcpe.ac.uk/policy-standards/pressuresacute-medical-specialities (accessed September 3rd 2015).

3. Liu SW, Singer SJ, Sun BC, Camargo CA. A Conceptual Model for Assessing Quality of Care for Patients Boarding in the Emergency Department: Structure-Process-Outcome. Acad Emerg Med. 2011; 18:430-5.

4. BBC News. 'Boarding' increases hospital stays and spread infections, researchers say. http://www.bbc.co.uk/news/ uk-scotland-23482451 (accessed November 19th 2015).

5. McKnight JA, Espie C. Managing acute medical admissions: the plight of the medical boarder. Scott Med J. 2012; 57:45-7.

6. Beckett D. Winter Pressures in NHS Scotland 2008-2009: Report for the Emergency Access Delivery Team. http://www. shiftingthebalance.scot.nhs.uk/initiatives/sbc-initiatives/emergencyaccess-delivery-programme/winter-planning/ (accessed September 3rd 2015).

7. British Medical Association. Beating the effects of winter pressures. https://www.bma.org.uk/news/2013/december/bma-plans-to-tacklewinter-pressures (accessed June 3rd 2016).

8. McMurdo MET, Witham MD. Unnecessary ward moves. Age Ageing [Online]. 2013;0:1-2. doi:10.1093/ageing/aft079. (accessed November 19th 2015).

9. The Scottish Government. Unscheduled Care. http://www.gov.scot/ Topics/Health/Quality-Improvement-Performance/UnscheduledCare (accessed November 19th 2015).

10. De Silva D. Improving patient flow across organisations and pathways. http://www.health.org.uk/sites/default/files/ ImprovingPatientFlowAcrossPathwaysAndOrganisations.pdf (accessed November 19th 2015).

11. Yap D, Macpherson S, Majumdar S. Boarder Patient - equal care from primary team?. http://attendee-io-production.s3.amazonaws. com/events/52c453cac76ab7b722000013/posters/ 8ab14ed3-56a7-4468-aa9a-4e6e5688f6cf/Boarder\%20Patient\% $20-\% 20$ equal\%20care\%20from\%20primary\%20team.pdf (accessed November 24th 2015). 